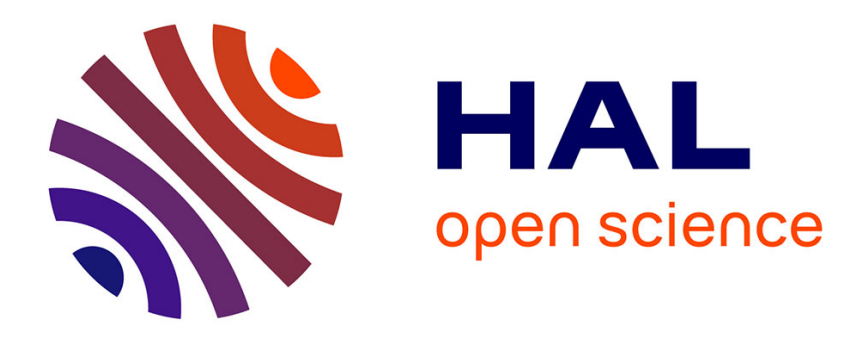

\title{
Spatial Locality Aware Disk Scheduling in Virtualized Environment
}

Xiao Ling, Shadi Ibrahim, Song Wu, Hai Jin

\section{To cite this version:}

Xiao Ling, Shadi Ibrahim, Song Wu, Hai Jin. Spatial Locality Aware Disk Scheduling in Virtualized Environment. IEEE Transactions on Parallel and Distributed Systems, 2015, pp.14. 10.1109/TPDS.2014.2355210 . hal-01087602

\section{HAL Id: hal-01087602 https://hal.inria.fr/hal-01087602}

Submitted on 28 Jun 2016

HAL is a multi-disciplinary open access archive for the deposit and dissemination of scientific research documents, whether they are published or not. The documents may come from teaching and research institutions in France or abroad, or from public or private research centers.
L'archive ouverte pluridisciplinaire HAL, est destinée au dépôt et à la diffusion de documents scientifiques de niveau recherche, publiés ou non, émanant des établissements d'enseignement et de recherche français ou étrangers, des laboratoires publics ou privés. 


\title{
Spatial Locality Aware Disk Scheduling in Virtualized Environment
}

\author{
Xiao Ling, Shadi Ibrahim, Song Wu, Member, IEEE, Hai Jin, Senior Member, IEEE
}

\begin{abstract}
Exploiting spatial locality, a key technique for improving disk I/O utilization and performance, faces additional challenges in the virtualized cloud because of the transparency feature of virtualization. This paper contributes a novel disk $1 / O$ scheduling framework, named Pregather, to improve disk I/O efficiency through exposure and exploitation of the special spatial locality in the virtualized environment, thereby improving the performance of disk-intensive applications without harming the transparency feature of virtualization. The key idea behind Pregather is to implement an intelligent model to predict the access regularity of spatial locality for each VM. Moreover, Pregather embraces an adaptive time slice allocation scheme to further reduce the resource contention and ensure fairness among VMs. We implement the Pregather disk scheduling framework and perform extensive experiments that involve multiple simultaneous applications of both synthetic benchmarks and MapReduce applications on Xen-based platforms. Our experiments demonstrate the accuracy of our prediction model and indicate that Pregather results in the high disk spatial locality and a significant improvement in disk throughput and application performance.
\end{abstract}

Index Terms-Virtualization, disk-intensive, l/O scheduling, spatial locality, efficiency

\section{INTRODUCTION}

Virtualization technology is extensively leveraged in cloud environments: it enables multiple virtual machines (VMs) - with multiple operating systems and applications - to run within a physical server. For example, Amazon web services [1] rely on the Xen virtualization hypervisor to provide the VM-based infrastructure as a service (IaaS) solution, which enables users to lease and customize their environments in order to run their applications. Virtualization however imposes new challenges in the scheduling and the allocation of system resources. With the volume of data growing rapidly and multiple disk intensive applications with different disk I/O characteristics (mixed applications) sharing an infrastructure [2], [3], allocating disk resources efficiently while guaranteeing VMs' I/O performance for preserving a high disk throughput becomes of key importance in virtualized environments.

Exploiting spatial locality is an important technique for scheduling I/O requests to improve disk I/O efficiency (i.e., high spatial locality results in a significant reduction in disk seek delay and rotation overhead, which leads to high disk throughput). For example, traditional file systems often allocate the accessed data of a process as contiguous blocks if possible, so disk scheduling can easily, according to I/O characteristics

- X. Ling, S. Wu and H. Jin is with the Services Computing Technology and System Lab, Cluster and Grid Computing Lab, School of Computer Science and Technology, Huazhong University of Science and Technology, Wuhan, 430074, China. Song $W u$ is the corresponding author. Email:wusong@hust.edu.cn.

- X. Ling is also with Information \& Communication Company of Hunan Electric Power Corporation of State Grid in China.

- S. Ibrahim is with Inria, Rennes Bretagne Atlantique Research Center, France. of the process, exploit spatial locality of requests. Unlike traditional environments, in a virtualized environment achieving high spatial locality is a challenging task, due to the transparency feature of virtualization which causes the semantic gap isolation between the hypervisor and guest VMs. As a result, when VMs with different disk intensive applications share disks in the cloud environment, the block I/O layer lacks a global view of the I/O access patterns of processes [4]. The lack of coordination between file systems in both the hypervisor and VMs reduces the efficiency of exploiting disk locality in virtualized environments. Moreover, a VM leased by users may encapsulates more than one application (e.g., multiple applications, including file-editing and media streaming, run in a virtual desktop; a Hadoop application generates multiple Map processes to parallel access data), which in turn increases the complexity and irregularity of the I/O behavior of the VM.

Research effort has been directed toward improving disk efficiency through exploiting spatial locality in virtualized environments. These efforts use either invasive mode scheduling (i.e., select the disk pair schedulers within both the hypervisor and the VM according to the applications' access patterns [5], [6]), or non-invasive mode scheduling (i.e., schedule the $\mathrm{I} / \mathrm{O}$ requests while treating a VM as a black box [7], [8]). However, the aforementioned solutions target similar types of applications (mainly read-dominated applications), and cannot be applied when a VM encapsulates mixed applications [4], [9]. Moreover, they come at the cost of violating the transparency feature of virtualization. Besides, although these solutions exploit spatial locality to improve disk $\mathrm{I} / \mathrm{O}$ utilization, they may not ensure I/O performance of each VM. For example, when some VMs deployed applications with low spatial locality and some VMs 
deployed applications with strong spatial locality run together, they prefer to serve applications with strong spatial locality with cost of the performance of the applications with low spatial locality. This may lead to starving some applications with low spatial locality and I/O contention among VMs.

This paper follows this line of research and contributes to the goal of improving throughput for complex I/O workloads, including write-dominated applications or mixed applications, by enabling efficient disk utilization and guaranteeing I/O performance of VMs, with preserving the transparency feature of virtualization. To end this, we solve two key issues: (1) detecting spatial locality in virtualized environments without any prior information of workloads; (2) making full use of spatial locality while guaranteeing I/O performance of each VM. In this paper we make the following three contributions to achieve this goal:

1) We investigate the spatial locality of disk data accesses in virtualized environments. By tracing the I/O requests of VMs with mixed applications, we observe that the disk data accesses are grouped into regions, bounded by the virtual disk sizes, and within each region the disk data accesses are grouped into sub-regions, which correspond to the applications' access patterns.

2) We introduce an intelligent prediction model that uses a temporal access-density clustering algorithm to analyze the data access of a VM with mixed applications. Our model can predict the distribution of sub-regions with spatial locality within each region and the arrival times of future requests accessing these sub-regions (i.e., detect the subregional spatial locality for each VM).

3) We propose Pregather, a disk scheduling framework with a spatial-locality-aware heuristic algorithm in the hypervisor for exploiting the special spatial locality (i.e., the regional and sub-regional spatial locality) to reduce disk seek and rotational overhead in the virtualized environment: Pregather does that - thanks to our prediction model - without any prior knowledge of the applications' access patterns. Besides, Pregather embraces an adaptive time slice allocation scheme based on the special spatial locality of VMs to further ensure fairness among VMs while improving disk I/O utilization.

We build the prototype of Pregather in Xen. Our evaluations, using synthetic benchmarks, a MapReduce application (distributed sort) and the database workloads, demonstrate the accuracy of the intelligent prediction model and the throughput benefits from our approach: Pregather achieves high disk spatial locality upon ensuring I/O performance of VMs, and thus improves the disk utilization and the applications' performance. For example, when multiple VMs with mixed applications runs together, in contrast to the default Xen disk I/O scheduler-Completely Fair Queuing (CFQ), Pregather achieves throughput performance improvement by a factor of $1.5 \mathrm{x}$ and improves I/O performance of applications.

The rest of the paper is organized as follows. Section 2 observes the disk access patterns in virtualized environments with mixed applications. Section 3 discusses our prediction model. Section 4 describes the design and implementation of Pregather. Section 5 details the performance evaluation. Section 6 discusses the related work. Finally, we conclude the paper in Section 7.

\section{Observing Disk access Patterns in VIRTUALIZED ENVIRONMENTS}

In this section, we seek to obtain an overview of understanding the spatial locality in virtualized environments. Ideally, we would like to get a rough idea of the disk access patterns in virtualized environments, and the impact of both virtualization features and mixed applications on the access patterns. So we experiment with two typical scenarios - typical synthetic benchmarks running on VMs and a MapReduce application running on a virtual cluster - and trace activities of $\mathrm{I} / \mathrm{O}$ requests in the two scenarios.

\subsection{Experimental Setup}

In the synthetic-benchmarks scenario, four guest VMs with different mixed $\mathrm{I} / \mathrm{O}$ workloads run in one physical node. Main features of access patterns of I/O workloads include read, write, sequential and random access. As shown in Table 1, we use sysbench [10] to generate these workloads with different disk access patterns and deploy them in different VMs.

In order to further observe disk access pattern in the real intensive-applications scenario, we deploy Hadoop (Hadoop-0.20.2 ${ }^{1}$ ) on a thirteen-nodes virtual cluster running on a three-nodes physical cluster, and run the sort benchmark ${ }^{2}$. Note that Hadoop with the sort benchmark is a typical and widely used example of the real I/O intensive and parallel applications. The access patterns of processes of sort benchmark are complicated due to the mixing of read, write, sequential and random access and the variable access intervals. We deploy five VMs in physical machine 1 (PM1), and four VMs in PM2 and PM3, respectively. In each PM, four VMs as data nodes owns eight map processes. The data set is 6GB for the sort benchmark (64MB block size).

In the two scenarios, the physical node is equipped with four quad-core $2.40 \mathrm{GHz}$ Xeon processor, 22GB of memory and one dedicated 1TB SATA disk of which availabe space is 100GB, running CentOS5 with kernel

1. Although the versions of Hadoop are changing, the Hadoop-0.20.2 is the most classical and stable version compared to other Hadoop versions. Besides, all Hadoop versions generate parallel processes (Map processes) to intensively access data and the types of their workloads are I/O intensive, such as sort, wordcount and so on.

2. In the sort benchmark, each mapper sorts the data locally, and each reducer merges the results from different mappers. And, the map input and the reduce output have the same data size as the input data. The I/O access patterns of processes are due to the sort benchmark and are not impacted by the changes of Hadoop versions 
TABLE 1: The description of the workloads running within VMs

\begin{tabular}{|c|l|l|}
\hline VM & Workload & Description \\
\hline \multirow{2}{*}{ VM1 } & Sequential Read-M $(S R-M)$ & 16 threads sequentially read 128 files, with total size of 1 GB \\
\cline { 2 - 3 } & Sequential Read-S(SR-S) & 16 threads sequentially read 1 file, with the size of 1 GB \\
\hline \multirow{2}{*}{ VM2 } & Sequential Write-M $(S W-M)$ & 16 threads sequentially write 128 files, with total size of 1 GB \\
\cline { 2 - 3 } & Sequential Write-S(SW-S) & 16 threads sequentially write 1 file, with the size of 1 GB \\
\hline \multirow{2}{*}{ VM3 } & Sequential Read-M $(S R-M)$ & 16 threads sequentially read 128 files, with total size of 1 GB \\
\cline { 2 - 3 } & Random Read \& Write $(R R W)$ & 16 threads randomly write and read 128 files, with total size of 1GB \\
\hline \multirow{2}{*}{ VM4 } & Random Read $(R R)$ & 16 threads randomly read 128 files, with total size of 1GB \\
\cline { 2 - 3 } & Random Write $(R W)$ & 16 threads randomly write 128 files, with total size of 1 GB \\
\hline
\end{tabular}

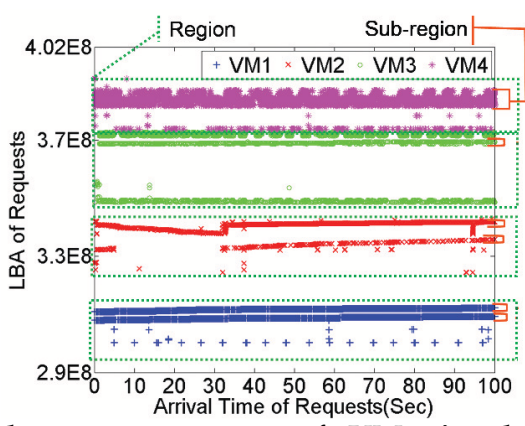

Fig. 1: Disk access patterns of VMs in the syntheticbenchmarks scenario

2.6.18. All results are obtained using Xen version 4.0.1 with Blktap AIO driver [11]. The guest VM is configured with two Virtual CPUs, 1GB memory and 12 GB virtual disk (the virtual disk is mapped to a default file-backed image). The default disk scheduler of VMs is Noop ${ }^{3}$ while the default disk scheduler of the hypervisor is the default CFQ. Besides, we use the blktrace tool [12] to track the logical block addresses (LBAs) and arrival times of requests from VMs.

\subsection{Synthetic-benchmarks Scenario}

Fig. 1 shows the change in the LBAs of arriving requests from four VMs in the synthetic-benchmarks scenario.

Our first observation is that the LBAs of requests from different VMs can be grouped into different regions which are occupied by VM images. The ranges [3.0012 $\times$ $\left.10^{8}-3.1739 \times 10^{8}\right]$, $\left[3.244 \times 10^{8}-3.426 \times 10^{8}\right],\left[3.4863 \times 10^{8}\right.$ $\left.3.727 \times 10^{8}\right]$ and $\left[3.738 \times 10^{8}-3.911 \times 10^{8}\right]$ represent the regions of VM1, VM2, VM3 and VM4, respectively. The size of each region is smaller than the 12GB size of VM image. The reason is that the file system of hypervisor assigns contiguous disk blocks to the $\mathrm{VM}$ image in order to improve disk efficiency. Accordingly, the VM has regional spatial locality when the average seek distance of a VM is smaller than the size of its image.

Our second observation is that looking at the disk accesses within each VM, LBAs and arrival times of requests divide the region of each $\mathrm{VM}$ into several sub-regions over time. These sub-regions differ in their ranges and access frequencies. The reason is that the

3. Noop has recently been used as the default VM scheduler because any (re)ordering at the VM level will be counterproductive as I/O requests from VMs will be dispatched to the disk device according to the disk scheduler at the hypervisor level. Thus, it is better to simply push the requests as early as possible, so as to save CPU cycles and avoid any conflict that could occur between the I/O schedulers at the hypervisor and VM levels.
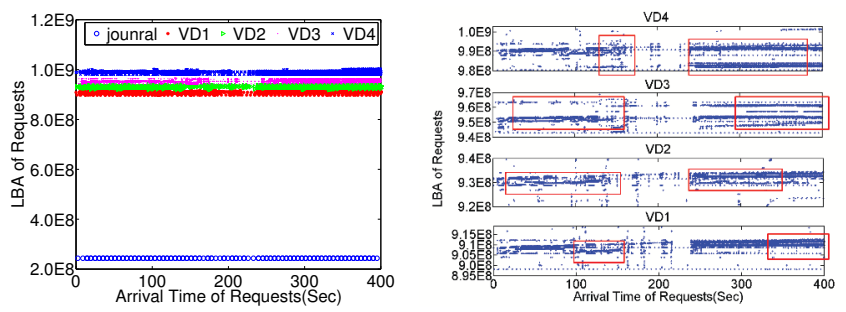

(a) The distribution of arriving (b) Zooming access patterns of each requests in disk of PM2

$\mathrm{VM}$ in PM2

Fig. 2: Disk access patterns of VMs in the MapReduceapplication scenario

file system of a VM also assigns contiguous virtual blocks to the applications without obtaining physical disk characteristics, and accordingly all VM image formats (e.g., RAW, Qcow [13], Fvd [14]) try to map LBAs of these virtual blocks into contiguous offsets in the VM image. As the hypervisor treats a VM as a process, the file system of the hypervisor maps the contiguous offsets into as contiguous LBAs as possible. Furthermore, because applications access their own data sub-regions, requests from the same application have sub-regional spatial locality, especially from applications with sequential access.

As shown in Fig. 1, these VMs have different characteristics of sub-regional spatial locality. This is because the features of access pattern of application impact on the sub-regional spatial locality of VMs. First, in contrast to VM1 and VM2, both VM3 and VM4 do not have a clear sub-regional spatial locality (i.e., the ranges of observed sub-regions are larger), because of the random access of data sets exhibited by random applications. For example, during 100s the requests from VM1 are mainly concentrated into two narrow sub-regions similar to thin horizontal lines (both ranges of sub-regions are less than $\left.1.4 \times 10^{6}\right)$. Instead, the most of the requests from VM4 are distributed in a large sub-region randomly: around $3.845 \times 10^{8}$ with a maximum distance of $\mp 2.7 \times 10^{6}$. Second, for VMs with write applications, the distribution of sub-regions and their ranges are more diverse. Moreover, the access frequencies of these sub-regions are often changing. For instance, the distribution and access frequencies of sub-regions of VM2 during 0 to 30s are different from the ones after 30s. This is due to a nondeterministic allocation of the write data set, the impact of disk cache, and update of inodes of files.

Fig. 1 also shows that some accessed sub-regions own the low access frequencies at the beginning of each region, thus not having spatial locality. This can be 
explained due to: (1) writing log files - the journal process in a VM periodically writes the system log files, the position of $\log$ files are normally at the start of the VM image; and (2) updating the metadata of the VM image, such as the access time and the changes of file content. The position of the inode is generally at the start of the VM image. Moreover, applications with write or random access introduce the frequent update of log files and the metadata, thus resulting in a growing number of sub-regions with low access frequencies, such as VM3 and VM4 in Fig. 1.

\subsection{MapReduce-application Scenario}

To prove our observations further, we track the information of arriving requests in the block layers of physical machines when running the sort benchmark. Fig. 2 shows the distribution of LBAs of arriving requests in disk of PM2 ${ }^{4}$ during 400s.

First, Fig. 2(a) describes that most of requests are from the four VMs (named VD1, VD2, VD3 and VD4, respectively) and a few requests are from journal process in hypervisor. These requests from different VMs are grouped into different regions in the MapReduce-application scenario as well as in the synthetic-benchmarks Scenario. For example, the LBAs of requests from VD1, VD2, VD3 and VD4 concentrate on the ranges $\left[8.981 \times 10^{8}-\right.$ $\left.9.194 \times 10^{8}\right],\left[9.202 \times 10^{8}-9.424 \times 10^{8}\right],\left[9.429 \times 10^{8}-9.688 \times\right.$ $\left.10^{8}\right]$ and $\left[9.803 \times 10^{8}-1.001 \times 10^{9}\right]$, respectively. Also, the sizes of these regions are smaller than the image file sizes of corresponding VMs. So VMs also own regional spatial locality in the MapReduce-application scenario. Besides, because the requests from journal process in the hypervisor access a fixed region periodically, this region does not have spatial locality.

Then, we amplify the regions corresponding to VMs with the I/O processes of Hadoop, in order to observe the sub-region spatial locality of VMs further. As shown in Fig. 2(b), within each region, LBAs of requests also concentrate different sub-regions with time. However, the distribution and ranges of VMs sub-regions are more variable and the number of sub-regions with low access frequency increases in the MapReduce-application scenario, compared to the synthetic-benchmarks scenario. The reason is that the VM as the date node of Hadoop encapsulates more than one I/O process whose access patterns interleave sequential and random access and are changing with time. These result in more complicated $\mathrm{I} / \mathrm{O}$ access patterns of VMs. Meanwhile, the increasing number of write operations and random access brings in frequently updating log file and metadata, which causes the increasing number of sub-regions with low access frequencies (e.g., VD1, VD3, and VD4).

Nevertheless, in some time periods each region also has some sub-regions which are similar to almost horizontal lines. As shown in Fig. 2(b), we use red rectangles

4. Because of limitation of pages, we show the distribution of LBA of requests in one of three physical machines. The observations of other two physical machines are same as that of PM2. to point out several horizontal lines within each VM's region during the time periods. This shows that a lot of requests in the time periods frequently access these subregions. Thus, the VMs with the MapReadue application own sub-regional spatial locality in these time periods. Moreover, the characteristics of sub-regional spatial locality of these VMs are changing with time. For instance, the distribution and access frequencies of sub-regions of VD2 during 0 to 50s are more diverse compared to ones during 50s to 100s. This is due to the change of access patterns of processes, especially write operations and random access. Furthermore, when the access patterns of some Hadoop's processes in a VM are sequential access, the thin lines exist within the region and the VM owns strong sub-regional spatial locality during these periods. For example, during a period from 20 s to 150 s, in spite of several sub-regions with low access frequencies or with larger ranges, the region of VD3 still owns two thin horizontal lines. This is because two processes with sequential access patterns access different data sets simultaneously although other processes randomly access data within the VD3's region during this period. Thus the regularity of sub-regional spatial locality of $\mathrm{VM}$ is changed based on access patterns of processes running in the VM.

\subsection{Discussion}

In summary, with respect to the cases when VMs with multiple processes run together, we observe the special spatial locality in virtualized environments: regional spatial locality across VMs and sub-regional spatial locality between requests from the same VM. Regional spatial locality is bounded by the size of VM image. Moreover, the subregional spatial locality of a VM is obvious when processes with sequential access run within a VM.

The virtualization transparency leads to inefficient exploitation of spatial locality and thus lowers disk utilization (i.e., increases disk head seeks (movement) between sub-regions); the traditional non-work-conserving schedulers, including CFQ [15] and Anticipatory scheduler (AS) [16], are effective at preserving the spatial locality exhibited by individual processes, but treat a VM process simply as a general user process and never recognize the sub-regional spatial locality of a VM, resulting in the low spatial locality (e.g., as shown in Section 5, the seek distance at zero is only $55 \%$ under CFQ in the syntheticbenchmarks scenario). The aim of our work is to make full use of the special spatial locality of VMs to improve physical disk efficiency and thus enhance the performance of applications in the virtualized environment.

According to above observations, our aim faces two mainly issues: (1) how to detect the regularity of the special spatial locality, especially sub-regional spatial locality, without any prior knowledge of applications; (2) how to maximize disk I/O utilization while guaranteeing I/O performance of VMs, especially when VMs with strong sub-regional spatial locality and VMs with weak sub-regional spatial locality share storage. 
TABLE 2: Variables of the vNavigator model

\begin{tabular}{|l|l||l|l|}
\hline Var. & Description & Var. & Description \\
\hline$V M$ & a guest VM & $\triangle n$ & the number of requests accessing $Z_{j}$ during interval $\left[T_{r}, T\right]$ \\
\hline$P(R)$ & the LBA of a request $R$ & $U_{i}$ & $\begin{array}{l}\text { the } i^{t h} \text { sub-region unit with sub-regional spatial locality of a VM in } \\
\text { current prediction window }\end{array}$ \\
\hline$T(R)$ & the arrival time of a request $R$ & $\lambda$ & a decay factor \\
\hline$B$ & the offset in the disk & $W\left(R_{j}, T\right)$ & the contribution of $R_{j}$ to spatial locality at prediction window $T$ \\
\hline$Z_{j}$ & the $j^{t h}$ equal-sized zone whose size is $B$ & $D\left(Z_{j}, T\right)$ & the temporal access-density of $Z_{j}$ at prediction window $T$ \\
\hline$R_{j}$ & a request accessing $Z_{j}$ & $\delta(V M, T)$ & the temporal access-density threshold of a VM at prediction window $T$ \\
\hline$R_{j}^{m}$ & the $m^{t} h$ request accessing $Z_{j}$ & $Z T\left(Z_{j}, T\left(R_{j}\right)\right.$ & the average access time interval of $Z_{j}$ when a request $R_{j}$ access $Z_{j}$ \\
\hline$T$ & the current prediction window & $S R\left(U_{i}\right)$ & the range of $U_{i}$ \\
\hline$T_{r}$ & a prediction window when $R_{j}$ arrives & $S T\left(U_{i}\right)$ & the future access interval of $U_{i}$ \\
\hline
\end{tabular}

\section{Prediction model of the Locality and REGULARITY OF DISK ACCESSES}

As discussed in Section 2, the regional spatial locality can be easily observed according to the VM image size. But the sub-regional spatial locality cannot be observed in the virtualized environment due to the virtualization transparency. Consequently, the disk scheduler in the hypervisor cannot efficiently exploit the sub-regional spatial locality of the VM. To this end, we design an intelligent prediction model, named vNavigator, to predict the regularity of the sub-regional spatial locality of a $\operatorname{VM}(i . e$. , the distribution of sub-regions with spatial locality and access intervals of these sub-regions). Hence the vNavigator model helps to guide I/O scheduling in the hypervisor. Based on the discussion about subregional spatial locality in Section 2, the design of vNavigator model faces three challenges: (1) the distribution of sub-regions with spatial locality is changing with time, and varies based on the access patterns of applications; (2) requests from background processes (named discrete requests) within a VM interfere with the prediction of future requests with sub-regional spatial locality; and (3) different sub-regions with spatial locality may have different access regularity. For clarity, Table 2 lists the variables used in the vNavigator model.

The vNavigator model uses a temporal access-density clustering (TAC) algorithm to analyze the historical data access within a VM image file to predict the future subregional spatial locality of the VM. As shown in Fig. 3 , considering the frequent changes in both the range and regularity of the sub-regions with spatial locality, the TAC algorithm divides the disk space into a series of equal-sized zones (denoted by $Z=\left\{Z_{1}, Z_{2}, \ldots, Z_{n}\right\}, n=$ $\left.\frac{\text { length }(\text { disk })}{B}\right)$. To capture and predict the change of the spatial locality in time, the TAC algorithm also divides the allocated serving time of a VM into several equal time windows (prediction window). By tracking the arrival time and the LBAs of VM's requests, the TAC algorithm quantizes the zones' access frequencies in previous windows to estimate the spatial locality of zones in the current prediction window.

Zones with possible spatial locality draw out the distribution of the sub-regions with relative spatial locality: given that zones with similar spatial locality may have different access frequencies and access intervals, the TAC algorithm therefore groups, within the same prediction window, neighboring zones into larger units (sub-region

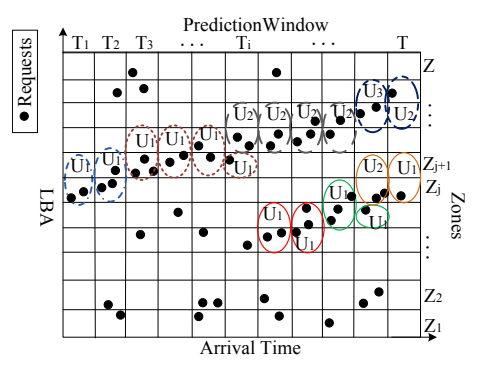

Fig. 3: vNavigator model: sub-region unit distribution

units). The distribution of sub-regions with spatial locality can be represented as $U=\left\{U_{1}, U_{2}, \ldots, U_{i}\right\}, U_{i} \subseteq$ $V M$, where $U_{i}$ represents the $i^{t h}$ sub-region unit with spatial locality. A sub-region unit may consist of one or two neighboring zones (further details are provided in the following subsections). Accordingly, the vNavigator model actually predicts the range of $U_{i}$ and the arrival time interval of future requests accessing $U_{i}$.

\subsection{Quantization of Access Frequency}

The TAC algorithm introduces a temporal access-density to quantize the access frequency of a zone. The temporal access-density of a zone is the sum of the contributions of historical requests to the future possibility of the zone's spatial locality in the current prediction window. Because the sub-regional spatial locality changes with time, the impact of recently arrived requests is greater than that of older requests on predicting sub-regional spatial locality. The contribution of historical requests therefore decline with time. Accordingly, we introduce an access weight of the request to represent the contribution of a request in the current prediction window. The access weight uses a decay factor $(\lambda)$ to quantize the relationship between the contribution of the request and time.

Definition 1: The access weight of a request $\left(R_{j}\right)$ to the spatial locality of a zone $\left(Z_{j}\right)$ in the current prediction window is:

$$
W\left(R_{j}, T\right)=\lambda^{-\left(T-T_{r}\right)}
$$

where $\lambda>1$ and $R_{j}$ accesses $Z_{j}$ in $T_{r}$.

According to (1), the access weight is 1 at the beginning, then decays toward zero with passing time. And the temporal access-density of the zone is defined as follows:

Definition 2: The temporal access-density of a zone $\left(Z_{j}\right)$ is the sum of the access weights of the requests accessing 
this zone in the current prediction window:

$$
D\left(Z_{j}, T\right)=\sum_{P(R) \in Z_{j}} W(R, T)
$$

where $R$ represents all requests accessing $Z_{j}$ until $T$.

According to (1) and (2), and to simplify the computational cost of the temporal access-density of a zone, we obtain the following Lemma 1.

Lemma 1: Given that $\Delta n$ is the number of arrived requests accessing $Z_{j}$ between $T_{r}$ and $T\left(T_{r}<T\right)$, $D\left(Z_{j}, T\right)$ is given by:

$$
D\left(Z_{j}, T\right)=\lambda^{-\left(T-T_{r}\right)} D\left(Z_{j}, T_{r}\right)+\triangle n .
$$

Proof: $n$ is the number of requests accessing $Z_{j}$ until $T$, and $R_{j}^{k}$ represents the $k^{t h}$ request to access $Z_{j}$.

$$
\begin{aligned}
D\left(Z_{j}, T\right) & =\sum_{k=1}^{n+\Delta n} W\left(R_{j}^{k}, T\right)=\sum_{k=1}^{n} W\left(R_{j}^{k}, T\right)+\sum_{k=n+1}^{n+\Delta n} W\left(R_{j}^{k}, T\right) \\
& =\lambda^{-\left(T-T_{r}\right)} \sum_{k=1}^{n} W\left(R_{j}^{k}, T_{r}\right)+\sum_{k=n+1}^{n+\Delta n} \lambda^{T-T} \\
& =\lambda^{-\left(T-T_{r}\right)} D\left(Z_{j}, T_{r}\right)+\triangle n
\end{aligned}
$$

According to Lemma 1, the temporal access-density consists of the number of newly arrived requests during the current prediction window and the decay of the temporal access-density of the previous prediction windows. Hence the temporal access-density captures that requests accessing a zone at different times have different effects on the prediction of the spatial locality of the zone.

\subsection{Explore Sub-regional Spatial Locality}

Depending on the temporal access-densities of the zones, we discuss the spatial locality of zones in the current prediction window. In a guest VM, the requests from background processes access some zones periodically. These zones do not have spatial locality, although their temporal access-densities are larger than zero. Therefore, the TAC algorithm uses a temporal access-density threshold to distinguish zones with future spatial locality. Considering the variations of the access frequencies of zones and interference of the system and journal processes in a VM, the temporal access-density threshold of a VM meet two conditions: (1) changing over time (i.e., when updating the temporal access-densities of zones); and (2) never dropping suddenly when increasing the number of zones with low temporal access-densities. Thus the temporal access-density threshold of a VM in the current prediction window can be stated as the mean of the accessed zones' temporal access-densities that are larger than 1. By excluding zones whose temporal accessdensities are lower than 1 , we reduce the impacts of the zones that were accessed a long time ago, and therefore avoid any sudden drop of the threshold.

Definition 3: The temporal access-density threshold of a $\mathrm{VM}(V M)$ in the current prediction window is:

$$
\delta(V M, T)=\sum_{y=1}^{N(T)} D\left(Z_{y}, T\right) / N(T)
$$

where $N(T)$ is the number of zones whose $D\left(Z_{y}, T\right) \geq 1$.

Based on the current temporal access-density threshold of the VM, we explore the possibility of sub-regional spatial locality of VM. When $D\left(Z_{j}, T\right)$ is larger than $\delta(V M, T)$, the access of data in $Z_{j}$ has sub-regional spatial locality in the next prediction window. Besides, the range of the sub-region with spatial locality may include zones with temporal access-densities more than $\delta(V M, T)$ and with temporal access-densities lower than $\delta(V M, T)$. For example, as shown in Fig. 3, in $T_{2}, D\left(Z_{j}\right.$, $\left.T_{2}\right)$ is larger than $\delta\left(V M, T_{2}\right)$ and $D\left(Z_{j+1}, T_{2}\right)$ is smaller than $\delta\left(V M, T_{2}\right)$, but $Z_{j+1}$ has sub-regional spatial locality in $T_{3}$. Therefore, when $D\left(Z_{j}, T\right)$ is larger than $\delta(V M, T)$, but the temporal access-density of $Z_{j+1}$ is smaller than $\delta(V M, T)$, the $T A C$ algorithm considers that both $Z_{j}$ and $Z_{j+1}{ }^{5}$ have sub-regional spatial locality of access in the next prediction window. Accordingly, the current distribution of the sub-regions with spatial locality consists of zones with higher temporal access-densities compared to the current temporal access-density threshold, and their neighbors with temporal access-densities lower than the current temporal access-density threshold.

\subsection{Access Regularity of Sub-regional Spatial Local- ity}

Given that different sub-regions have different access regularity and different zones may have different access regularity, we introduce a sub-region unit which comprises one or two neighboring zones with the same access regularity and relative spatial locality. Therefore, the range of a sub-region unit is defined as follows, in accordance with the above subsection on exploring subregional spatial locality:

Definition 4: The range of a sub-region unit in the current distribution of sub-regions with spatial locality of the VM is:

$$
S R\left(U_{i}\right)=\left\{\begin{array}{c}
\left(Z_{j}, Z_{j+1}\right) \\
D\left(Z_{j}, T\right) \geq \delta(V M, T), D\left(Z_{j+1}, T\right)<\delta(V M, T) \\
Z_{j} ; \\
D\left(Z_{j}, T\right) \geq \delta(V M, T), D\left(Z_{j+1}, T\right) \geq \delta(V M, T)
\end{array}\right.
$$

where $Z_{j+1}$ belongs to other sub-region units with relative spatial locality when $D\left(Z_{j+1}, T\right) \geq \delta(V M, T)$.

According to Definition 4, the sub-region unit with spatial locality includes one zone whose temporal accessdensity is more than the temporal access-density threshold of the VM, and its neighbor with temporal accessdensity lower than this threshold. This allows us to gather arrival intervals between historical requests with relative sub-regional spatial locality to predict the arrival time interval of future requests. To reduce the cost of the model and remove the interference of discrete requests, we use the average access time interval of the zone with temporal access-density more than the threshold, in order to estimate the access time interval of the corresponding sub-region unit.

Definition 5: The future access interval of a sub-region unit with sub-regional spatial locality is:

$$
S T\left(U_{i}\right)=Z T\left(Z_{j}, T\left(R_{j}^{m}\right)\right), D\left(Z_{j}, T\right) \geq \delta(V M, T)
$$

5. $Z_{j-1}$ is not included due to the following reasons :(1) reducing disk backward seek and rotation overheads; and (2) avoiding overlapping zones. 
where $Z T\left(Z_{j}, T\left(R_{j}^{m}\right)\right)$ is the average access interval of $Z_{j}$ when $R_{j}^{m}$ access $Z_{j}$, and is denoted by:

$Z T\left(Z_{j}, T\left(R_{j}^{m}\right)\right)=\frac{Z T\left(Z_{j}, T\left(R_{j}^{m-1}\right)\right) *(m-1)+T\left(R_{j}^{m}\right)-T\left(R_{j}^{m-1}\right)}{m}$

\section{Disk Scheduling Based on Special SPATIAL LOCALITY}

Our aim is to exploit the special spatial locality of VM to improve the performance of mixed applications and disk I/O efficiency in virtualized environments while preserving virtualization transparency. Given that the nonwork-conserving mode is good at exploiting spatial locality - it prefers to wait for incoming requests whose LBAs are closest to the position of disk head rather than dispatching pending requests and therefore avoid the disk seek overhead - and based on the observations in Section 2, our design needs to answer two critical questions: (1) whether to wait for future request and how long is the waiting time when being ready to dispatch request; (2) how to maximize disk I/O utilization while guaranteeing I/O performance of VMs, especially when VMs with strong sub-regional spatial locality and VMs with weak sub-regional spatial locality share storage.

We design and implement an adaptive non-workconserving disk scheduling framework with a spatiallocality-aware (SPLA) heuristic algorithm in the hypervisor, named Pregather. The SPLA heuristic algorithm takes advantage of the regional spatial locality across VMs and the sub-regional spatial locality prediction of the vNavigator model, to guide Pregather to make the decision on waiting for future requests. Besides, based on the special spatial locality and the I/O characteristics of VMs, Pregather allocates the dynamic I/O service time to each VM, to reduce I/O contention among VMs while improving the disk $\mathrm{I} / \mathrm{O}$ utilization.

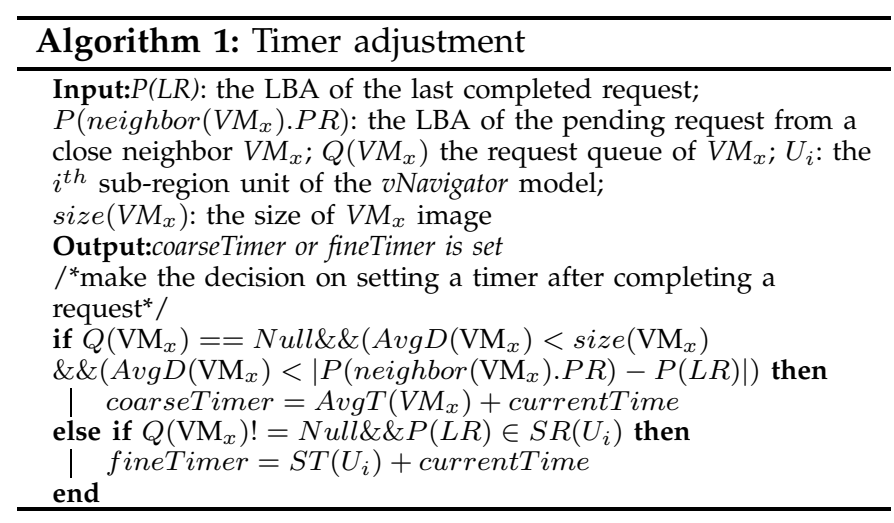

\subsection{Spatial-locality-aware Heuristic Algorithm}

The key function of the SPLA heuristic algorithm is to evaluate the relationship between the cost of waiting for future requests and the cost of disk seeking for serving the pending request whose LBA is close to the position of disk head. To end this, the SPLA heuristic algorithm works considering the position of the disk head, the

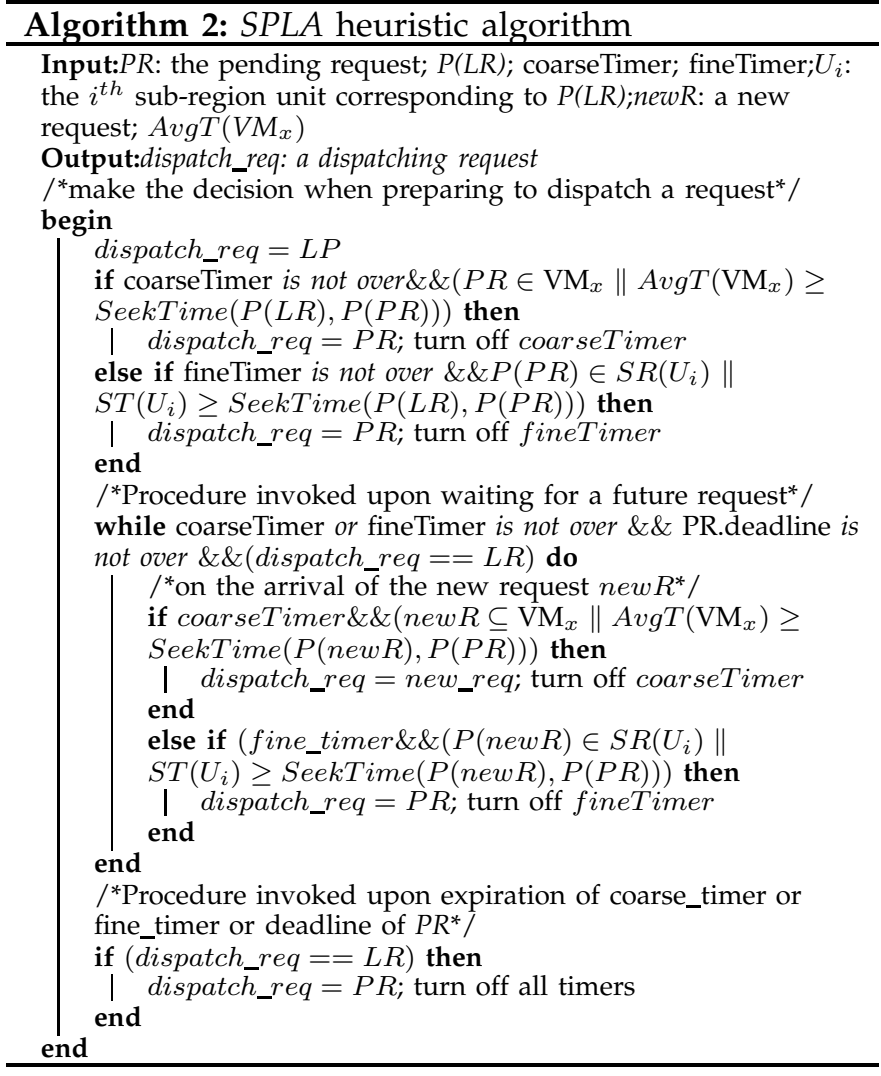

access regularity of the regional and sub-regional spatial locality of VMs, and the pending requests.

After completing a request (i.e., $L R$ ) from the current served $\mathrm{VM}^{6}$ (i.e., $\left.V M_{x}\right)$, Pregather introduces a timer to wait for future requests with special spatial locality (shown in Algorithm 1). According to the observations in Section 2, when all pending requests are not from the current served VM, exploiting the regional spatial locality can lower disk seek overheads across VMs. When some pending requests are from the current serving VM, exploiting the sub-regional spatial locality can reduce the disk seek overhead between sub-regions. So Pregather uses two types of timers - a coarse waiting time for future requests with regional spatial locality and a fine waiting time for future requests with subregional spatial locality - and then chooses the timer based on the position of the disk head and the current pending requests. If the hypervisor does not have any pending requests from $V M_{x}$ after dispatching $L R$, Pregather considers whether to set the coarse waiting time: if the average distance between LBAs of requests from $V M_{x}$ (i.e., $\operatorname{Avg} D\left(V M_{x}\right)$ ) is smaller than the size of $\mathrm{VM}$ image and the distance between the LBAs of $L R$ and the pending request from a close neighbor $\mathrm{VM}$, the coarse waiting time is set to the average arrival interval of $V M_{x}$ (i.e., $\left.\operatorname{Avg} T\left(V M_{x}\right)\right)$. Instead, if $V M_{x}$ has pending requests, Pregather decides whether to set the fine waiting time

6. The LBA of a completed request is the position of disk head at this time 
and consults the vNavigator model to predict the subregional spatial locality around the disk-head position. If the model predicts that the position of $P(L R)$ is in the range of a sub-region unit with spatial locality (i.e., $S R\left(U_{i}\right)$ ), the fine waiting time is set to the average access interval of the sub-region unit (i.e., $\left.S T\left(U_{i}\right)\right)$.

When the physical block device driver prepares to dispatch a request, as shown in Algorithm 2, Pregather selects the closest pending request (i.e., $P R$ ) to $L R$ according to the LBAs. Pregather estimates the disk seek time between the LBAs of $L R$ and $P R$ (SeekTime $(P(L R)$, $P(P R))$ ). When the coarse waiting time is set, if $P R$ meets one of the following two conditions: either the estimated seek time between the request and $P(L R)$ is smaller than $\operatorname{Avg} T\left(V M_{x}\right)$; or the request is from $V M_{x}$, the algorithm decides to dispatch $P R$. Otherwise, the algorithm decides to wait for a new request that meets one of these two conditions. On the other hand, when the fine waiting time is set, if LBA of $P R$ also belongs to $S R\left(U_{i}\right)$, or SeekTime $(P(L R), P(P R))$ is smaller than $S T\left(U_{i}\right)$, the algorithm decides to dispatch $P R$. Otherwise, the algorithm waits for a new request whose LBA belongs to $S R\left(U_{i}\right)$ or estimated seek time is smaller than $S T\left(U_{i}\right)$. Once the timer or the deadline of $P R$ is overtime, Pregather dispatches $P R$ to avoid from starving any requests.

\subsection{Adaptive Time Slice Allocation among VMs}

When VMs with strong special spatial locality and VMs with weak special spatial locality are run together, the SPLA heuristic algorithm, as it mainly focusses on exploiting special locality, speed up I/O performance of VMs with strong spatial locality at cost of the performance of VM with weak special spatial locality. This may lead to unfairness among VMs. Some schedulers rely on static time slice allocation for VMs, such CFQ combined with Blkio-Cgroup [17], and therefore ensure performance isolation and fairness among VMs. As discussed in [18], the actual bandwidth requirement of a workload is strongly related to the spatial locality and the size of request. These solutions based on the static time slice allocation don't consider the I/O characteristics of VMs, resulting in a waste of the disk I/O bandwidth). Accordingly, Pregather uses an adaptive I/O time slice allocation scheme for serving VMs and therefore avoids disk I/O contention and unfairness among VMs while keeping the efficiency of the SPLA heuristic algorithm.

The adaptive time slice allocation scheme initially assigns an equal time slice (e.g., $\left.T S_{x}^{0}\right)$ to each VM, then adjusts the length of the time slice dynamically according to the spatial locality of VMs. In the virtualized environment, the size of requests is limited and always smaller than $44 \mathrm{~KB}$. So we ignore the impact of the request size on the time slice allocation. To quantize the special spatial locality of VM, we introduce a Regional spatial locality Indicator $\left(R I\right.$, e.g. $\left.R I_{x}\right)$ and a Sub-regional spatial locality Indicator (SI, e.g., $S I_{x}$ ) for each VM. The RI of a VM is stated as: $R I_{x}=\operatorname{Avg} D\left(V M_{x}\right) / \operatorname{Sizeof}(V M)$, where

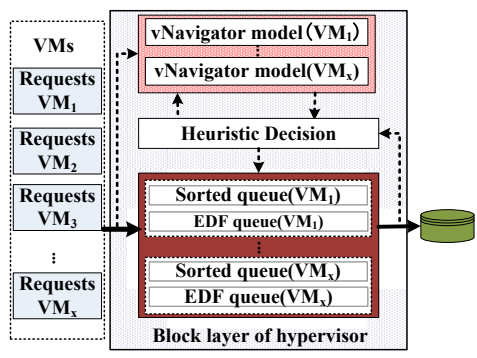

Fig. 4: Architecture of Pregather

$\operatorname{Avg} D\left(V M_{x}\right)$ is the average distance between LBAs of requests from VM and Sizeof $\left(V M_{x}\right)$ is the size of the VM image. The SI of a VM is the percentile of the disk seek distance between dispatched requests of VM at zero. When $R I_{x}$ is larger than 1 , this means that the VM does not own the regional spatial locality. So the length of the initial time slice is reduced, which leaves more time to serve the VMs with regional spatial locality. When $R I_{x}$ is smaller than 1 then if $S I_{x}$ is higher than $55 \%$, the VM has clear sub-regional spatial locality and the length of the initial time slice of the VM is increased.

In the basis of the changes of both indicators, the length of the time slice of a VM (e.g., $\left.T S_{x}\right)$ is changing as follows:

$$
T S_{x}=\left\{\begin{aligned}
T S_{x}^{0} \times\left(S I_{x}-55 \%\right) \times 10 ; \quad 0<R I_{x} \leq 1, S I_{x}>55 \% \\
T S_{x}^{0} ; \quad 0<R I_{x} \leq 1, S I_{x} \leq 55 \% \\
T S_{x}^{0} / 2 ; \quad R I_{x}>1
\end{aligned}\right.
$$

According to (8), if $R I_{x}$ is larger than 1, the length of time slice of $V M_{x}$ is set to half of the length of the initial time slice, in order to avoid from wasting of disk $\mathrm{I} / \mathrm{O}$ bandwidth while ensure the performance of this VM. Otherwise, if $S I_{x}$ is smaller than $55 \%$, the length of the time slice of $V M_{x}$ is kept as the initial time slice to guarantee the performance of VMs with week subregional spatial locality. If $S I_{x}$ is larger than $55 \%$, the length of the time slice of $\mathrm{VM}$ is increased with the growth of the $S I_{x}$, to maximize the disk I/O utilization.

Furthermore, when the block layer does not have pending requests from a served VM and the time slice of the VM is not used up, the length of the time slice of the VM is reduced by half. This means that the type of requests from the served $\mathrm{VM}$ is not $\mathrm{I} / \mathrm{O}$ intensive and Pregather gives more resources to VM with I/O intensive.

\subsection{Implementation}

We implement a prototype of Pregather in the Xenhosted platform. As shown in Fig. 4, Pregather consists of the vNavigator models corresponding to $\mathrm{VMs}$, and the heuristic decision module that implements the SPLA heuristic algorithm, at the block layer of the hypervisor. To make full use of the special spatial locality without starving requests, Pregather, similar to CFQ, builds two types of request queues for each VM: a sorted queue and an earlier deadline first (EDF) queue. The sorted queue contains requests in the order of their LBAs, while the EDF queue contains requests in the order of their deadline (the deadline value of Pregather is the same as of 
CFQ). Also, Pregather allocates each VM a dynamic I/O time slice and serves VMs in a round robin fashion, to reduce I/O interference among $\mathrm{VMs}$ and batch as many requests from the same $\mathrm{VM}$ as possible. The round robin fashion decides the service order of VMs based on the distance between the position of VM and the position of disk head .

When a new request from a VM arrives in the hypervisor, Pregather assigns the request a deadline and queues the request in both the sort queue and EDF queue. Then Pregather calculate the average arrival time and the average distance between LBAs of requests from the VM, and updates the RI of the VM. Meanwhile, Pregather triggers the corresponding vNavigator model to analyze and update the range and arrival time interval of the sub-region units immediately.

After completing a request from the current served $\mathrm{VM}$, the heuristic decision module sets a coarse timer or a fine timer based on the average arrival time and average distance of the VM and the vNavigator model. When the hypervisor prepares to dispatch a request, Pregather selects a pending request (named $P R$ ) before triggering the heuristic decision module. If the request queues of the current served VM are empty, Pregather selects a request from a VM whose location is close to the current served VM (in the disk). Otherwise, Pregather first checks the deadline of the head request in the EDF queue of the current served VM. If the deadline of the head request has expired, Pregather dispatches this request immediately. If not, Pregather selects a request next to the last completed request in the sorted queue of this VM as $P R$. Then, if the timer is active, the heuristic decision module decides whether to dispatch $P R$, without exceeding the deadline of $P R$. Once the heuristic decision module schedules a future request, Pregather maintains the idle state of the disk head until the arrival of a suitable request. If the timer runs out or the deadline of $P R$ expires, Pregather dispatches $P R$.

Besides, after dispatching a request, Pregather computes the disk seek distance between the current dispatched request and the last dispatched request from the same VM. Then, Pregather updates the SI of the VM based on the disk seek distance. According to the change of both RI and SI and the number of pending requests of the VM, Pregather triggers the adaptive time slice allocation scheme to adjust the length of the time slice of the VM.

It is important to note that Pregather is not limited to Xen and can be implemented in other hypervisors (e.g., KVM [19] and Linux-VServer [20]). The vNavigator model (introduced in section IV) uses hypervisorindependent parameters including the arrival times and LBAs of requests. Also, the historical decision module is implemented as a separate module at the block I/O layer of the hypervisor. Moreover, the vNavigator model can be applied in other physical block devices (e.g., multiple disks, SSD), because the prediction of the model is not impacted by the physical characteristics of disks.

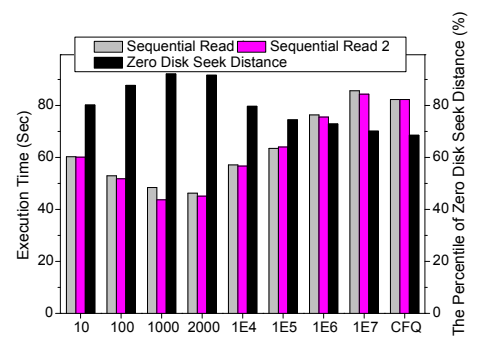

Fig. 5: The execution time of two sequential read applications and the proportion of seek distance at zero point under Pregather with different offsets, CFQ

\section{Performance Evaluation}

We run a suite of experiments evaluating our Pregather on the Xen-hosted platform using synthetic benchmarks, a MapReduce application and the database application. The first set of experiments is to verify the vNavigator model, including evaluation of the sensitive parameter and verification of its accuracy. The second set of experiments is to evaluate the overall performance of Pregather when there are multiple VMs with different access patterns. The third set of experiments is to evaluate the overheads caused by Pregather. The experimental setup is the same that described as in Section 2.1.

\subsection{Verification of vNavigator Model}

An accurate sub-regional spatial locality prediction is a key factor to achieve the high disk utilization with Pregather: Pregather uses the sub-regional spatial locality prediction of the vNavigator model to schedule requests. Hence we evaluate the accuracy of the prediction model. The impact of $B$ value. On the basis of the design of vNavigator model, the offset $B$ of the model impacts the temporal access-densities of zones and the accuracy of clustering zones with spatial locality. Accordingly, to define a suitable $B$, we run two sequential read applications (16 threads sequentially read 128 files, whose total size is 2GB) in a VM, and capture their performance variation when changing $B$ (we fix $\lambda$ at 2, and the prediction window size to $20 \mathrm{~ms}$ ). The higher the accuracy of the prediction of the vNavigator model, the lower the frequency of disk seeking, because Pregather waits for a suitable future request with a minimal (zero) seek distance according to the vNavigator model. Thus we track the disk seek distance to discuss the prediction of the vNavigator model.

Fig. 5 shows the execution time of the two applications and the proportion of the disk seek distance at zero under different $B$ values $(B=1$ represents the size of a sector, i.e., 512bytes). Increasing $B$ from 10 to 1000 reduces the execution time of applications and increases the proportion of minimal seek distance. The reason for this is that small $B$ leads to the temporal access-densities of all zones tending toward the same value. The vNavigator model treats a zone accessed by a background process as a zone with spatial locality, thus introducing unnecessary waiting, especially when $B$ is equal to the size of the request. On the other hand, 
TABLE 3: The Execution time of applications running on a VM

\begin{tabular}{|l|l|l|l|l|}
\hline VM & Workload & Pregather & CFQ & AS \\
\hline SRRR & Sequential Read-M & $26.92 \mathrm{~s}$ & $44.0 \mathrm{~s}$ & $45.83 \mathrm{~s}$ \\
\cline { 2 - 5 } VM & Random Read & $33.74 \mathrm{~s}$ & $44.75 \mathrm{~s}$ & 45.94 \\
\hline RRRR & Random Read & $101.17 \mathrm{~s}$ & $119.18 \mathrm{~s}$ & $113.18 \mathrm{~s}$ \\
\cline { 2 - 5 } VM & Random Read & $108.29 \mathrm{~s}$ & $119.18 \mathrm{~s}$ & $112.39 \mathrm{~s}$ \\
\hline $\begin{array}{l}\text { SWSW } \\
\text { VM }\end{array}$ & $\begin{array}{l}\text { Sequential Write- } \\
\text { M }\end{array}$ & $27.27 \mathrm{~s}$ & $40.07 \mathrm{~s}$ & $39.66 \mathrm{~s}$ \\
\cline { 2 - 5 } & $\begin{array}{l}\text { Sequential Write- } \\
\text { M }\end{array}$ & $28.04 \mathrm{~s}$ & $42.72 \mathrm{~s}$ & $39.35 \mathrm{~s}$ \\
\hline $\begin{array}{l}\text { SWRW } \\
\text { VM }\end{array}$ & $\begin{array}{l}\text { Sequential Write- } \\
\text { M }\end{array}$ & $15.04 \mathrm{~s}$ & $25.79 \mathrm{~s}$ & $26.07 \mathrm{~s}$ \\
\cline { 2 - 5 } & Random Write & $47.06 \mathrm{~s}$ & $53.64 \mathrm{~s}$ & $53.05 \mathrm{~s}$ \\
\hline $\begin{array}{l}\text { RWRW } \\
\text { VM }\end{array}$ & Random Write & $57.56 \mathrm{~s}$ & $66.39 \mathrm{~s}$ & $66.63 \mathrm{~s}$ \\
\cline { 2 - 5 } & Random Write & $57.56 \mathrm{~s}$ & $65.81 \mathrm{~s}$ & $66.63 \mathrm{~s}$ \\
\hline SWRR & Sequential Write- & $58.76 \mathrm{~s}$ & $81.10 \mathrm{~s}$ & $85.92 \mathrm{~s}$ \\
VM & M & & & \\
\cline { 2 - 5 } & Random Read & $81.78 \mathrm{~s}$ & $89.50 \mathrm{~s}$ & $88.80 \mathrm{~s}$ \\
\hline $\begin{array}{l}\text { SRRW } \\
\text { VM }\end{array}$ & Sequential Read-M & $33.03 \mathrm{~s}$ & $44.06 \mathrm{~s}$ & $50.03 \mathrm{~s}$ \\
\cline { 2 - 5 } & Random write & $49.98 \mathrm{~s}$ & $52.75 \mathrm{~s}$ & $56.23 \mathrm{~s}$ \\
\hline
\end{tabular}

when increasing $B$ from 1000 to 2000, Pregather slightly decreases the average execution time of applications by $2 \%$, but maintains $91.6 \%$ of the seek distance at zero. This is explained that the number of zones at 1000 is more than at 2000, introducing more time overheads when updating the temporal access-densities of zones. However, with increasing B from 2000 to $10^{7}$, the execution time of both applications increases from $46 \mathrm{~s}$ to $85 \mathrm{~s}$, while the proportion of seek distance at zero drops from $91.6 \%$ to $71.2 \%$. The performance of Pregather at $10^{7}$ is the same as that of CFQ. This is because the size of the zone may cover the complete disk region taken by the VM image with increasing $B$, which cause that our model cannot detect the possible sub-regional spatial locality. Therefore, $B$ is restricted to the range between the size of the request and the size of the VM image.

The ratio of successful waiting. Based on the above discussion, we set $B$ to 2000 . Then, in seven different scenarios with different disk access patterns, we compare the performance of mixed applications within a VM under Pregather, CFQ and AS, to discuss the effectiveness of the model. Table 3 shows the execution time of the applications described in Table 1 . The performance of applications under Pregather is better than under CFQ and AS. In particular, Pregather outperforms CFQ and AS by $33 \%$ and $31 \%$, respectively, for the sequential write applications in the SWSW VM. By recording the activity of timer, we find that Pregather achieves a 90.6\% success ratio on waiting for a suitable future request, and therefore reduces the seek time. In contrast, CFQ and AS treat the VM as a general process and thus never wait for the future request, although they also employ the non-work-conserving mode.

Moreover, the vNavigator model also captures the spatial locality of access between applications when sequential applications are mixed with random applications, and consequently improves the performance of the applications, as in SRRR VM, SWRW VM, SWRR VM, and $S R R W$ VM. For instance, in SRRR VM, Pregather reduces the execution time of sequential read and random read by $38 \%$ and $22 \%$, respectively, compared with CFQ
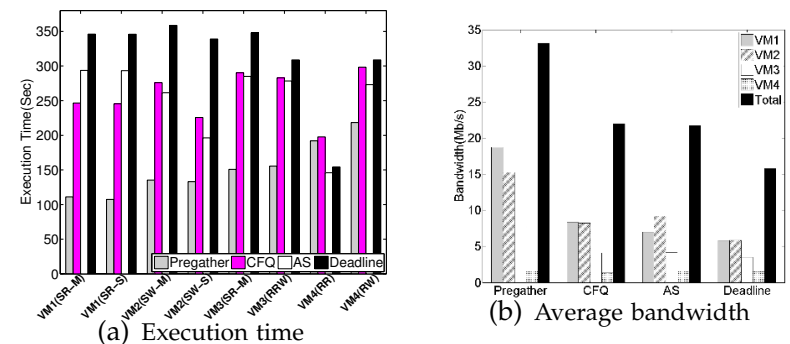

Fig. 6: The performance of mixed applications when four VMs run together

TABLE 4: The distribution of disk seek distance when four VMs run together

\begin{tabular}{|l|l|l|l|l|}
\hline Distance & Pregather & CFQ & AS & Deadline \\
\hline 0 & $75.78 \%$ & $55.06 \%$ & $49.97 \%$ & $12.54 \%$ \\
\hline$[-2.4 \mathrm{E} 7,2.4 \mathrm{E} 7]$ & $97.39 \%$ & $96.67 \%$ & $96.57 \%$ & $70.99 \%$ \\
\hline $\begin{array}{l}\text { [-1E9,-2.4E7] } \\
{[2.4 \mathrm{E} 7,1 \mathrm{E} 9]}\end{array}$ & $2.61 \%$ & $3.37 \%$ & $3.43 \%$ & $29.01 \%$ \\
\hline
\end{tabular}

and AS. In SWRR VM, Pregather outperforms CFQ for sequential write and random read applications by $27 \%$ and 9\%, respectively. On the other hand, with Pregather, the improvement of random applications in RWRW VM and in $R R R R \mathrm{VM}$ is $12 \%$ and $10 \%$, respectively. This is because the access pattern of random applications leads to the weak sub-regional spatial locality for a VM, as discussed in Section 2. Fortunately, the vNavigator model still prevents the interference of requests from background processes of a VM, and achieves an $80.4 \%$ success ratio on waiting for a suitable future request.

\subsection{Spatial-Locality-Aware Disk Scheduling for Mul- tiple VMs}

We design experiments to measure the efficiency of Pregather for exploiting both the regional and sub-regional spatial locality (the initial I/O time slice for each VM is set to 200ms). Therefore, we compare the performance of multiple VMs under Pregather with that under three schedulers of the Xen-hosted platform (CFQ, AS and Deadline). Unlike CFQ and AS, Deadline is a workconserving scheduler that dispatches neighboring requests without waiting for suitable future requests.

\subsubsection{Mixed Applications with Different Access Patterns}

We evaluate the performance of Pregather for the different mixed applications in the synthetic-benchmarks scenario described in Section 2.1. Fig. 6 shows the performance of mixed applications and VMs under four schedulers. Compared with CFQ, AS and Deadline, Pregather improves the bandwidth of VMs and performance of applications, especially VMs with sequential applications. The performance of Deadline as the representative of work-conserving mode is worse than other schedulers. Although both CFQ and AS use the nonworking conserving mode to batch requests from the same VM, they do not achieve the high performance of applications because of ignoring the sub-regional spatial locality. For example, as shown in Fig. 6(a), for VM1 with different sequential applications, Pregather reduces the 


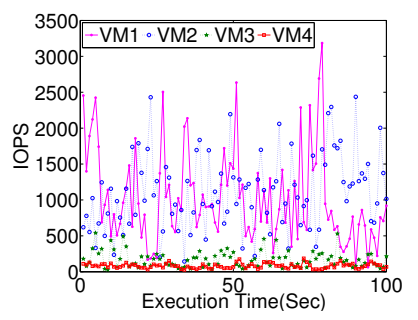

(a) Pregather

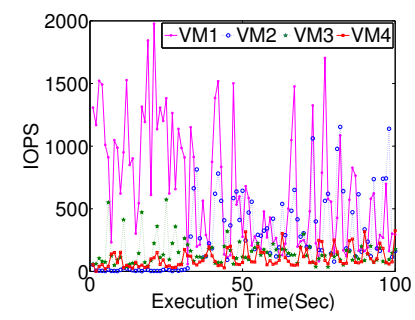

(b) CFQ

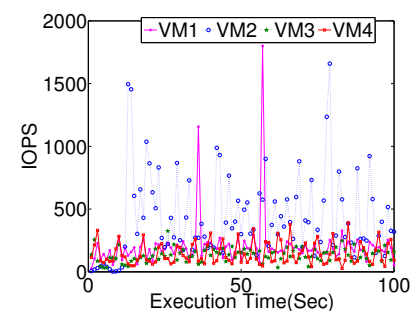

(c) AS

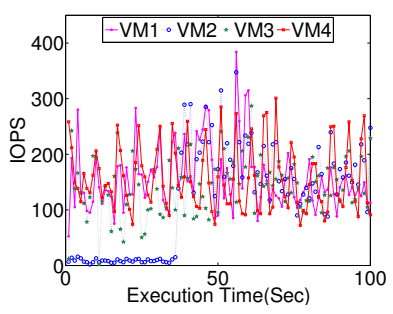

(d) Deadline

Fig. 7: The change of throughput of VMs when four VMs with mixed applications run together

execution time of both $S R-M$ and $S R-S$ applications by $55 \%, 62 \%$ and $68 \%$ in comparison with CFQ, AS and Deadline, respectively. Besides, the sub-regional spatial locality of VM3 is weaker than VM1 and VM2 as discussed in Section 2.2. Fortunately, Pregather boosts the performances of $S R-M$ application and $R R W$ application by more than $50 \%$ and more than $44 \%$ compared to the other schedulers. This is due to Pregather maximizing sub-regional spatial locality of VM3 in the I/O time slice of VM3. In addition, because of the limitation of physical bandwidth and the seeking overhead of the random applications, Pregather only improves the performance of the $R R$ application and $R W$ application in VM4 by $3 \%$ and $26 \%$ in comparison with CFQ. Although Deadline and AS outperform Pregather for the RR application in VM4, they sacrifice the performance of other VMs.

Fig. 6(b) also shows that Pregather improves the total bandwidth by $1.5 x, 1.6 x$ and $2.2 x$ compared with CFQ, AS and Deadline, respectively. The VM4's bandwidth under Pregather is almost the same as that under Deadline and AS while the other VMs' bandwidth under Pregather is higher than the other schedulers. This is because (1)Pregather with the SPLA heuristic algorithm exploits the special spatial locality of the VM in a given time slice of the VM to improve disk I/O efficiency, (2)Pregather allocates the adaptive time slices to VMs based on the VMs' spatial locality, to ensure I/O performance of VMs.

Disk seek overheads. To further illustrate disk I/O efficiency with Pregather, Table 4 presents the distribution of the disk seek distance under four schedulers. The seek distance with Pregather, CFQ and AS is mainly concentrated in the range from $-2.4 \times 10^{7}$ to $2.4 \times 10^{7}$, because the VM image occupies $2.4 \times 10^{7}$ sectors and the three schedulers exploit regional spatial locality across VMs to batch the requests from the same VMs. Besides, the proportion of disk seek distance at zero point with Pregather is $75.78 \%$, higher than the other schedulers, because the SPLA heuristic algorithm for each VM continuously dispatches requests with the subregional spatial locality by waiting for future requests successfully. When the newly dispatched request and the last dispatched request are from different VMs between which the distance is far, or the newly dispatched request is from the journal process of the hypervisor, the seek distance is distributed in the range between $-1 \times 10^{9}$ and $-2.4 \times 10^{7}$ or from $2.4 \times 10^{7}$ to $1 \times 10^{9}$.

Time slice allocation among VMs. To discuss the effi- ciency of the adaptive time slice allocation in Pregather, Fig. 7 shows the changes in throughput of four VMs during 100s. As shown in Fig. 7(a), under Pregather, VMs achieve different throughput due to different degrees of their sub-regional spatial locality. For example, the throughput of both VM1 and VM2 is higher than that of VM3 and VM4, because VM1 and VM2 have stronger sub-regional spatial locality than VM3 and VM4. Meanwhile, although the sub-regional spatial locality of VM4 is weakest, the minimal throughput of VM4 is still more than 70IOPS. Besides, the throughput of the VM2 often changes. For instance, the trend of VM2's throughput during 0 to $30 \mathrm{~s}$ is different from its after 30s. This is because write operations lead to the changing subregional spatial locality and thus Pregahter adjusts the time slice of VM2 dynamically.

In contrast, Fig. 7(b) demonstrates that under CFQ, VM2 has slightly starved in the beginning when VM1 grabs a lot of bandwidth. Also, CFQ allocates a static and equal time slice to each VM and serves VMs in a round robin fashion, therefore it cannot guarantee the I/O performance of each VM and wastes the disk $\mathrm{I} / \mathrm{O}$ resource due to ignoring the characteristics and the access patterns of VMs. Moreover, because both AS and Deadline neither assign a given I/O time slice to each $\mathrm{VM}$ nor serve $\mathrm{VM}$ in the round robin fashion, as shown in the Fig. 7(c) and Fig. 7(d), the I/O interference among VMs is more serious and the throughput of VMs under AS and Deadline is lower than that under Pregather.

\subsubsection{Real I/O Intensive Applications}

To discuss the performance of Pregather for real I/O intensive applications, we test a Hadoop with sort benchmark under Pregather, CFQ, AS, and Deadline in two different scenarios: with and without a background VM hosting the database applications (TPC-H).

The experimental setup of the first scenario is described in Section 2.1. Fig. 8 shows the execution time and average bandwidth of each physical machine under the four schedulers. As shown in Fig. 8(b), Pregather improves the total bandwidth of the physical cluster by $26 \%, 28 \%$, and $38 \%$ compared with CFQ, AS, and Deadline, respectively. Consequently, Pregather outperforms CFQ by $18 \%$ and AS by $20 \%$ for the sort benchmark. To discuss disk I/O efficiency further, Fig. 9 illustrates distribution of the seek distance in physical machines. Because Deadline sends as many adjacent requests as 


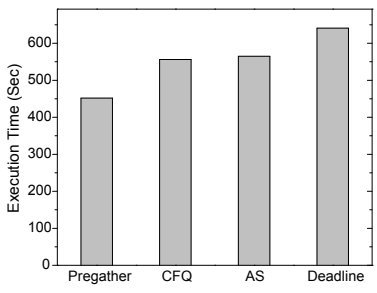

(a) Execution time

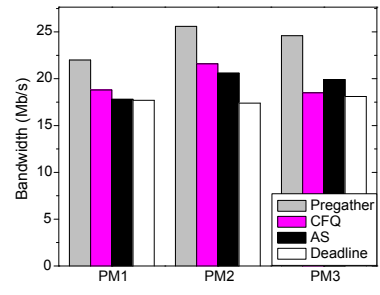

(b) Average bandwidth
Fig. 8: The performance and throughput of VMs when running sort benchmark

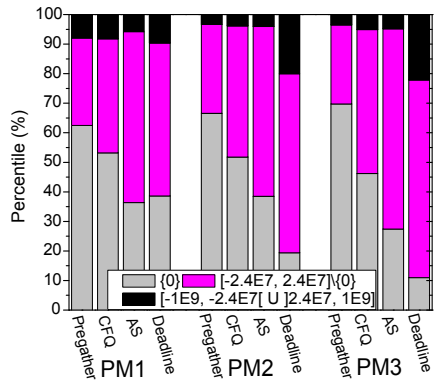

Fig. 9: The distribution of seek distance of PMs when running sort benchmark

possible without waiting for future requests, the seek overhead under Deadline is higher than that under the other schedulers, especially in PM2 and PM3 where only data nodes run. Although the distribution of the seek distance mainly concentrates on the space range of the image with Pregather, CFQ and AS for exploiting the spatial locality among VMs, the proportion of seek distances at zero with Pregather is much higher than with CFQ and AS. This is explained that the parallel processes of the sort benchmark sequentially access data sets during some periods, and thus leads to the clear sub-regional spatial locality of VMs (data nodes). Pregather captures these $\mathrm{VMs}^{\prime}$ sub-regional spatial locality to reduce the disk seek overhead with the SPLA heuristic algorithm.

In the second scenario, we deploy a VM (named TPCH VM) with two different TPC-H instances (q6 and q19) and a five-nodes virtual cluster with Hadoop on a physical node. In the virtual cluster, we generate a 2GB data set in four VMs (named Data VM) each of which has two maps. Fig. 12 shows the execution time of the applications and the analysis of seek distance under Pregather, CFQ, AS and Deadline. We observe that Pregather improves the performance of three applications in contrast to $\mathrm{CFQ}$ and AS. For instance, compared with CFQ, Pregather reduces the execution time of sort benchmarks $20 \%$ because of capturing the clear subregional spatial locality of Data VMs. Meanwhile, Pregather only improves the performance of $\mathrm{q} 6$ and $\mathrm{q} 9$ by $9 \%$ and $4 \%$, because the TPC- $\mathrm{H}$ instance accesses the data set randomly and TPCH VM own weak sub-regional spatial locality. Deadline costs the performance of Data VMs although improving the performance of $\mathrm{TPCH}$ VM. Besides, the proportion of minimal seek distance with Pregather is 65\% and higher than that with other schedulers as shown in 10(b).

Furthermore, Fig. 11 describes the changes of Data

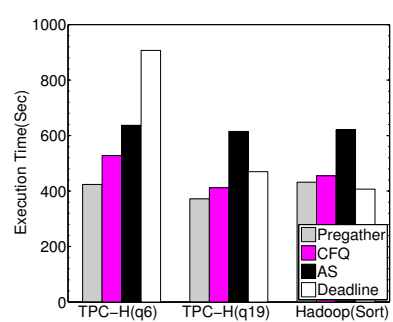

(a) Execution time

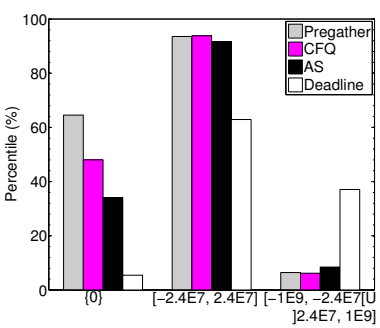

(b) The distribution of seek distance
Fig. 10: The performance and distribution of seek distance when the sort benchmark and TPC-H are running together

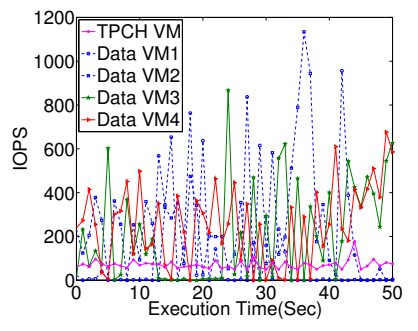

(a) Under Pregather

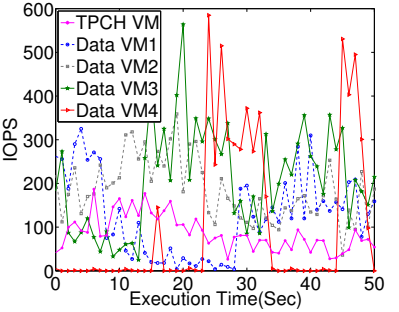

(b) Under CFQ
Fig. 11: The changes of throughput of Data VMs and TPC-H VM

$\mathrm{VMs}^{\prime}$ and TPCH VM's throughput under Pregather and CFQ during 100s, to discuss the time slice allocation of Pregather for the real and complicated I/O applications. As shown in Fig. 11(a), the changes of throughput in Data VMs are sharp, but their average throughput still achieves 300IOPS. Although the access patterns of processes and the arrival intervals of requests are variable in the sort benchmark as discussed in Section 2.3, Pregather can perceive the changes of sub-regional spatial locality of these VMs and then dynamically adjust the Data VMs' time slices. Besides, although the sub-regional spatial locality of TPCH VM is weaker, Pregather still maintains around 80IOPS the throughput of TPCH VM. In contrast, the TPCH VM grabs the bandwidth of Data VMs under CFQ. For example, as illustrated in Fig. 11(b), the highest throughput of TPC-H VM achieves 180IOPS and the average throughput is around 90IOPS. However, the average throughput of Data VMs with strong spatial locality are only 200IOPS and their jitter is serious.The reason is that CFQ neglects different degree of $\mathrm{VMs}^{\prime}$ spatial locality and allocates the same time slice to VMs.

\subsection{Overheads of Pregather}

According to the implementation of Pregather, it stores the historical information of the accessed zones including the the temporal access-density, the number of arrived requests, the total arrival time interval and so on. Meanwhile, Pregather calculates the average distance of LBAs and access time interval between requests from the same VM, the temporal access-density, the average access interval of zones, and so on. Therefore, Pregather may cause the extra memory and CPU overheads. To evaluate these overheads, we compare the free memory and CPU utilization of the hypervisor under Preagher with those under CFQ in the synthetic-benchmarks sce- 


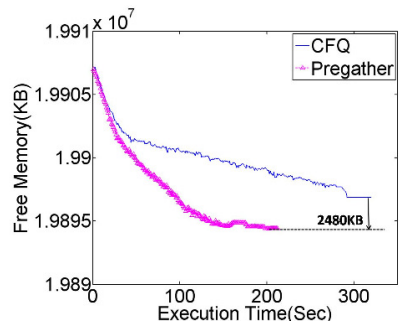

(a) Free memory

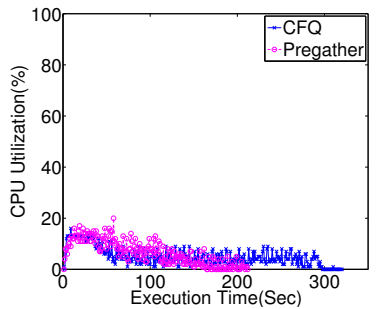

(b) CPU utilization
Fig. 12: The memory and CPU overheads of hypervisor under Pregather and CFQ

nario. This is because CFQ stores and compuates the historical access information of VMs to predict the spatial locality of process.

As shown in Fig. 12(a), with the arrival of requests from VMs, the rate of decline of free memory under Pregather is faster than under CFQ. Compared with CFQ, Pregather uses less than $2148 \mathrm{~KB}$ additional free memory. In contrast to the total free memory, the memory overheads caused by Pregather can be neglected. Fig. 12(b) shows that the highest CPU utilization under Pregather is no more than $20 \%$ and under CFQ achieves $17 \%$. Pregather only introduces $3 \%$ of the extra CPU overheads compared to CFQ. Moreover, CPU utilization under Pregather and under CFQ are great low. So the I/O intensive applications do not spend CPU and the CPU power does not affect the performance of I/O applications, which are also discussed by Kundu et al [21].

\section{Related Work}

Ever since the advent of virtualization technology, a huge number of studies have been dedicated to improving the performance of disk-intensive applications in virtualized environments [4]-[9], [22]. On the one hand, some of these solutions use invasive mode scheduling to manage I/O requests [5], [6], [22]. They introduce an additional hypervisor-to-VM interface to achieve better coordination between the disk scheduler within both the hypervisor and VMs. However, these solutions can only be applied when VMs are running the same type of application. Moreover, they require the hypervisor to be aware of the applications running within VMs, which harms the transparency feature of virtualization.

On the other hand, some solutions use non-invasive mode scheduling to manage the $\mathrm{I} / \mathrm{O}$ requests in virtualized environments without harming the transparency feature of virtualization [4], [7]-[9]. Streaming scheduling (SS) [9] turns any work-conserving disk scheduler into a non-work-conserving one based only on the request's own locality, thus reducing the disk seek time. SS essentially examines the existence of a stream by analyzing the characteristics of requests with relative spatial locality. Antfarm [4] enables the hypervisor to track the creation and exits of processes in VMs to infer the information of processes. The process information can help the disk scheduler at the hypervisor level to check the existence of read streams and map requests at the right read stream.
However, both SS and Antfarm can only infer read streams, and cannot be applied for write applications or mixed applications running within a VM.

Besides, these solutions improve disk I/O throughput via capturing the regularity of read applications with strong spatial locality and speeding up their I/O performance. However, when multiple VMs with different applications run together, these solutions result in the disk resource contention among VMs and cannot guarantee I/O performance of VMs.

To the best of our knowledge, analyzing the access regularity of VMs has thus far been performed only when a specific (one) application is running within a VM (such as online transaction processing, mail server or file migration) [23]-[26]. Moreover, exploring the benefits of predicting the access regularity of processes to exploit the disk spatial locality (e.g., by facilitating I/O perfecting) has so far been discussed only in nonvirtualized environments [27]-[29]. These studies cannot be applied in virtualized environments, because unlike general processes, the access patterns of VM processes are more complicated and variable. Our work focuses on investigating and exploiting the disk access locality and regularity in virtualized environments when mixed applications are running within each VM. As far as we know, we are the first to explore the benefits of the locality and regularity of data accesses to improve the disk efficiency in the presence of mixed applications while preserving the transparency feature of virtualization and ensuring I/O performance of VMs.

\section{Conclusion}

In this study, we investigate the disk access patterns of VMs encapsulating mixed applications. Our studies reveal that disk accesses can be grouped into regions bounded by the virtual disks sizes, and within each region the disk accesses are grouped into sub-regions, which correspond to the applications' access patterns. Ignoring the special spatial locality (i.e., the regional and sub-regional spatial locality) when scheduling I/O requests causes the performance degradation due to the high seek delay and the rotation overhead. We address this issue by developing Pregather, a new spatial-localityaware disk scheduler that exploits the special spatial locality for improving disk-intensive applications. Pregather embraces an intelligent prediction model, named vNavigator, to predict the distribution of sub-regions within each region, and the arrival times of future requests accessing these sub-regions. We perform extensive experiments that involve multiple simultaneous applications of both synthetic benchmarks and a MapReduce application on Xen-based platforms. Our experiments show the accuracy of our prediction model and indicate that Pregather results in the high spatial locality and a significant improvement in disk throughput.

Regarding future work, to alleviate the lower spatial locality that occurs in the presence of disk fragmentswe 
intend to extend Pregather to enable an intelligent allocation of physical blocks. Also, we are interested in applying Pregather in the data store environments consisting of multiple disks to improve I/O performance of VMs.

\section{ACKNOWLEDGMENTS}

The research is supported by National Science Foundation of China under grant No.61472151 and No.61232008, National 863 Hi-Tech Research and Development Program under grant No.2013AA01A208, Chinese Universities Scientific Fund under grant No.2013TS094, Research Fund for the Doctoral Program of MOE under grant No.20110142130005 and the ANR MapReduce grant (ANR-10-SEGI-001).

\section{References}

[1] Amazon Web Services. http://aws.amazon.com/.

[2] M. Armbrust, A. Fox, R. Griffith, A. Joseph, R. Katz, A. Konwinski, G. Lee, D. Patterson, A. Rabkin, I. Stoica, and M. Zaharia, "A view of cloud computing," Commun. ACM, vol. 53, no. 4, pp. 50-58, 2010.

[3] D. Le, H. Huang, and H. Wang, "Understanding performance implications of nested file systems in a virtualized environment," in Proc. FAST'12, 2012, pp. 8-8.

[4] S. Jones, A. Arpaci-Dusseau, and R. Arpaci-Dusseau, "Antfarm: Tracking processes in a virtual machine environment," in Proc. ATC'06, 2006, pp. 1-14.

[5] S. Ibrahim, H. Jin, L. Lu, B. He, and S. Wu, "Adaptive disk I/O scheduling for mapreduce in virtualized environment," in Proc. ICPP'11, 2011, pp. 335-344.

[6] M. Kesavan, A. Gavrilovska, and K. Schwan, “On disk I/O scheduling in virtual machines," in Proc. WIOV'10, 2010, pp. 6-6.

[7] S. Seelam and P. Teller, "Virtual I/O scheduler: a scheduler of schedulers for performance virtualization," in Proc. VEE'07, 2007, pp. 105-115.

[8] X. Ling, H. Jin, S. Ibrahim, W. Cao, and S. Wu, "Efficient disk I/O scheduling with qos guarantee for xen-based hosting platforms," in Proc. CCGrid'12, 2012, pp. 81-89.

[9] Y. $\mathrm{Xu}$ and S. Jiang, "A scheduling framework that makes any disk schedulers non-work-conserving solely based on request characteristics," in Proc. FAST'11, 2011, pp. 9-9.

[10] A. Kopytov, "Sysbench manual," http://sysbench.sourceforge.net/docs/.

[11] P. Barham, B. Dragovic, K. Fraser, S. Hand, T. Harris, A. Ho, R. Neugebauer, I. Pratt, and A. Warfield, "Xen and the art of virtualization," ACM SIGOPS Oper. Syst. Rev., vol. 37, no. 5, pp. 164-177, 2003.

[12] J. Axboe and A. D. Brunelle, "Blktrace User Guide," http://www.cse.unsw.edu.au/aaronc/iosched/doc/blktrace.html.

[13] The QCOW Image Format. http://people.gnome.org/ markmc/ qcow-image-format-version-1.html.

[14] C. Tang, "FVD: a high-performance virtualmachine image format for cloud," in Proc. USENIX ATC'11, 2011, pp. 18-18.

[15] J. Axboe. Completely Fair Queuing (CFQ). http://en.wikipedia.org/wiki/CFQ, 2010.

[16] S. Iyer and P. Druschel, "Anticipatory scheduling: A disk scheduling framework to overcome deceptive idleness in synchronous I/O," ACM SIGOPS Oper. Syst. Rev., vol. 35, no. 5, pp. 117-130, 2001.

[17] blkio-contoller. https://www.kernel.org/doc/Documentation/ cgroups/blkio-controller.txt.

[18] X. Zhang, K. Davis, and S. Jiang, "Qos support for end users of i/o-intensive applications using shared storage systems," in Proceedings of 2011 International Conference for High Performance Computing, Networking, Storage and Analysis. ACM, 2011, p. 18.

[19] KVM. http://www.linux-kvm.org/page/mainpage.

[20] Linux VServer. http://linux-vserver.org/Documentation, 2010.

[21] S. Kundu, R. Rangaswami, A. Gulati, M. Zhao, and K. Dutta, "Modeling virtualized applications using machine learning techniques," Proc. of VEE'12, vol. 47, no. 7, pp. 3-14, 2012.
[22] D. Boutcher and A. Chandra, "Does virtualization make disk scheduling passé?" ACM SIGOPS Oper. Syst. Rev., vol. 44, no. 1, pp. 20-24, Mar. 2010.

[23] Y. Hu, X. Long, and J. Zhang, "I/O behavior characterizing and predicting of virtualization workloads," Journal of Computers, vol. 7, no. 7, pp. 1712-1725, 2012.

[24] I. Ahmad, "Easy and efficient disk I/O workload characterization in vmware esx server," in Proc. IISWC'07, 2007, pp. 149-158.

[25] I. Ahmad, J. Anderson, A. Holler, R. Kambo, and V. Makhija, "An analysis of disk performance in VMware ESX server virtual machines," in Proc. WWC'03, 2003, pp. 65-76.

[26] A. Gulati, C. Kumar, and I. Ahmad, "Storage workload characterization and consolidation in virtualized environments," in Proc. VPACT'09, 2009.

[27] N. Tran and D. Reed, "Automatic arima time series modeling for adaptive I/O prefetching," IEEE TPDS, vol. 15, no. 4, pp. 362-377, 2004.

[28] X. Ding, S. Jiang, F. Chen, K. Davis, and X. Zhang, "Diskseen: exploiting disk layout and access history to enhance I/O prefetch," in Proc. USENIX ATC'07, 2007, pp. 1-14.

[29] Z. Li, Z. Chen, S. Srinivasan, and Y. Zhou, "C-miner: Mining block correlations in storage systems," in Proc. FAST'04, vol. 186, 2004.

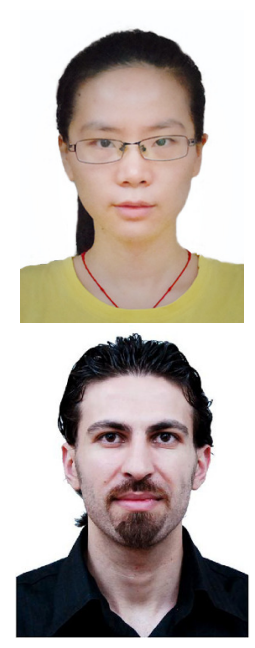

Xiao Ling is an engineer at Information \& Communication Company of Hunan Electric Power Corporation of State Grid in China. She received her B.S. (2008) degree at National University of Defense Technology, and M.S. (2011) and Ph.D. (2014) degree at Huazhong University of Science and Technology. Her research interests are in the area of cloud computing and virtualization, focusing on virtualized I/O optimization in cloud.

Shadi Ibrahim is a researcher within the KerData team at INRIA Rennes, working on scalable distributed data processing in the Cloud He holds a Ph.D. in Computer Science from Huazhong University of Science and Technology (HUST) in China. He has several years of experience with academic research at HUST, INRIA research center and Microsoft research center in Asia. His research interests include cloud computing, data-intensive computing, virtualization technology, file and storage systems.

Song Wu is a professor of computer science and engineering at Huazhong University of Science and Technology (HUST) in China. He received his Ph.D. from HUST in 2003. He is now the director of Parallel and Distributed Computing Institute at HUST. He is also served as the vice director of Service Computing Technology and System Lab (SCTS) and Cluster and Grid Computing Lab (CGCL) of HUST. His current research interests include grid/cloud computing

and virtualization technology.
Hai Jin is a Cheung Kung Scholars Chair Professor of computer science and engineering at Huazhong University of Science and Technology (HUST) in China. He is now Dean of the School of Computer Science and Technology at HUST. Jin received his $\mathrm{PhD}$ in computer engineering from HUST in 1994. In 1996, he was awarded a German Academic Exchange Service fellowship to visit the Technical University of Chemnitz in Germany. Jin worked at The University of Hong Kong between 1998 and 2000, and as a visiting scholar at the University of Southern California between 1999 and 2000. $\mathrm{He}$ was awarded Excellent Youth Award from the National Science Foundation of China in 2001. Jin is the chief scientist of ChinaGrid, the largest grid computing project in China, and the chief scientist of National 973 Basic Research Program Project of Virtualization Technology of Computing System. Jin is a senior member of the IEEE and a member of the ACM. Jin is the member of Grid Forum Steering Group (GFSG). He has co-authored 15 books and published over 400 research papers. His research interests include computer architecture, virtualization technology, cluster computing and grid computing, peerto-peer computing, network storage, and network security. 